\title{
Fuzzy Logic Controller Based Distributed Generation Integration Strategy for Stochastic Performance Improvement
}

\author{
Jagdish Prasad Sharma $^{1}$ and H. Ravishankar Kamath ${ }^{2}$ \\ ${ }^{1}$ Mewar University, Chittorgarh, Rajasthan, India \\ ${ }^{2}$ Malwa Institute of Technology, Indore, India \\ Correspondence should be addressed to Jagdish Prasad Sharma; jpsharma.cseb@gmail.com
}

Received 15 July 2016; Accepted 12 October 2016

Academic Editor: Mamun B. Ibne Reaz

Copyright (c) 2016 J. P. Sharma and H. R. Kamath. This is an open access article distributed under the Creative Commons Attribution License, which permits unrestricted use, distribution, and reproduction in any medium, provided the original work is properly cited.

\begin{abstract}
In the restructured environment, distributed generation (DG) is considered as a very promising option due to a high initial capital cost of conventional plants, environmental concerns, and power shortage. Apart from the above, distributed generation (DG) has also abilities to improve performance of feeder. Most of the distribution feeders have radial structure, which compel to observe the impact of distributed generations on feeder performance, having different characteristics and composition of time varying static ZIP load models. Two fuzzy-based expert system is proposed for selecting and ranking the most appropriated periods to an integration of distributed generations with a feeder. Madami type fuzzy logic controller was developed for sizing of distributed generation, whereas Sugeno type fuzzy logic controller was developed for the DG location. Input parameters for Madami fuzzy logic controller are substation reserve capacity, feeder power loss to load ratio, voltage unbalance, and apparent power imbalances. DG output, survivability index, and node distance from substation are chosen as input to Sugeno type fuzzy logic controller. The stochastic performance of proposed fuzzy expert systems was evaluated on a modified IEEE 37 node test feeder with 15 minutes characteristics time interval varying static ZIP load models.
\end{abstract}

\section{Introduction}

In the restructured environment, feasibility study of distributed generation (DG) integration to existing grid is a key interesting area of research. Deployment of distributed generation (DG) in distribution system brings technical as well as financial benefits to utilities. The positive benefits are loss reduction, reliability enhancement, and power quality improvement and negative effects are increased fault level and false operation of the feeder. Most of the distribution feeders have radial structure and are designed to operate with a single source along the feeder. To compensate rapid growth of load demand and proliferation of electronic device, available options for utilities are network extension, substation capacity augmentation, and DG integration. Apart from the above, the majority of the existing radial distribution feeders are lengthy, over and nonuniform loading, which resulted in an excessive voltage drop, poor voltage profile, male tripping of protection devices, and power loss. DG integration to existing grid is well suited option to utilities due to lack of financial resources and long-term implementation of grid extension work. As DG installation greatly influences the performance of distribution feeder, the optimal location and sizing of distributed generation (DG) are an active research interest, which is needed to harness maximum benefits from the DG

Several researchers have used different optimization methods such as analytical, numerical, and heuristic for the sake of power loss minimization, cost reduction, profit maximization, and environmental emission reduction. Barin et al. have used a fuzzy-based expert system for choosing and ranking of the most appropriated periods to integrate distribution generation with an existing distribution network [1]. A fuzzy-based power management technique is employed to schedule power dispatching for microgrid/utility grid. The proposed power management technique is subjected to a set of constraints, including weather conditions, load-shedding hours, and peak pricing hours [2]. In order to mitigate 
demand and avoid power outage in peak hour, two fuzzy logic controller are simulated for optimal location and sizing of distributed generation on IEEE 13 test feeder [3].

A fuzzy logic method is deployed for optimal DG placement subjected to minimize total power loss constraint, whereas a new analytical method is used for DG sizing. The effectiveness of DG on system voltage profile and branch power losses is carried out on IEEE 69 and IEEE 33 radial feeder [4]. Manjili and Rajaee proposed fuzzy controller for energy management and cost reduction microgrid. The amount of power exchange from storage unit is based on the load demand, renewable generation rate, and electricity price [5].

Metia and Ghosh. presented optimal location of DG units with a 33-bus system based on the available amount of DG using fuzzy logic [6]. A firefly based algorithm for optimal location and capacity of CHP technology DG or a photovoltaic DG is implemented on IEEE 37-node feeder with the objectives of profit maximization [7]. Harmony search algorithm is utilized to determine appropriate size of shunt capacitors with real power losses and installation cost of shunt capacitors, whereas the location of shunt capacitors is identified using voltage stability index [8]. Padma Lalitha et al. presented a fuzzy approach for finding optimal DG locations and a PSO algorithm for optimal DG sizes on IEEE 33 node feeder [9]. A probabilistic fuzzy solution is proposed to identify vulnerable nodes for the optimal reconfiguration problem [10]. Fuzzy expert system employed for optimal capacitor placement and sizing for 35 buses with multilevel of loads [11].

Arabali et al. carried out a stochastic framework to optimal sizing and reliability analysis for a hybrid power system having wind power, photovoltaic (PV), and energy storage system. The stochastic nature of wind, solar irradiation, and photovoltaic (PV) power and load are stochastically modelled using ARMA [12]. Soroudi and Ehsan investigated the impact of an uncertain power production of distributed generations (DGs) on active losses of distribution feeder. Uncertainty in wind speed and gas turbines is modelled by a Weibull probability distribution function (PDF) and fuzzy, respectively [13].

Sharma and Ravishankar Kamath have presented voltage assessment indices for modified IEEE 37 node test feeder having time varying composite voltage sensitive load [14]. Performance indices to assess feeder performance of modified IEEE 37 node test feeder were developed with the help of forward-backward sweep method and two port parameters representation of feeder components [15].

The objective of this paper is to integrate DG using two fuzzy logic controllers. One fuzzy logic controller is used to determine the sizing of DG on the basis of feeder performance parameter such as substation reserve capacity, feeder power loss to load ratio, voltage unbalance, and apparent power imbalance. Another fuzzy logic controller is used to choose the DG location node on the basis of DG output; survivability index and node distance from substation are chosen as input.

\section{Feeder Performance Indices}

The quality of power supply for modified IEEE 37 node test feeder is evaluated to develop performance indices and these performance indices are substation reserve capacity, voltage unbalance factor, and feeder power loss to load ratio, branch loading, voltage deviation, and power factor [15]. It is observed that the said substation transformer of feeder is highly overloaded between 35 and 71 characteristics time interval, which could be relived with solar PV penetration at the feeder. Modified feeder has also accounted for stochastic characteristics and composition of voltage sensitive load models. These load models are categorized into residential, commercial, and industrial consumers. Each category consumers are appliances with constant power, constant current, and constant impedance in random propositions. The participation of real and reactive power load exponents for the different type categories of consumers is characterized by the following equations [15]:

$$
\begin{aligned}
P_{a b c}^{k}(h)= & C 1_{a b c}^{k}(h)+C 2_{a b c}^{k}(h) *\left|\frac{V_{a b c}^{k}(h)}{V N_{a b c}}\right|^{2} \\
& +C 3_{a b c}^{k}(h) *\left|\frac{V_{a b c}^{k}(h)}{V N_{a b c}}\right|, \\
Q_{a b c}^{k}(h)= & D 1_{a b c}^{k}(h)+D 2_{a b c}^{k}(h) *\left|\frac{V_{a b c}^{k}(h)}{V N_{a b c}}\right|^{2} \\
& +D 3_{a b c}^{k}(h) *\left|\frac{V_{a b c}^{k}(h)}{V N_{a b c}}\right| .
\end{aligned}
$$

Calculation of ZIP loads compositions is shown in Appendix. To meet smart grid implementation criterion: performance indices are evaluated for 15 minutes characteristics time interval for the whole day. In these days, distribution generation integration is a common practice to meet out continuously increased demand. The load and renewable DG generation probabilistic nature are considered in this study. It is observed from load flow solution that feeder is subjected to overloading during $8 \mathrm{AM}$ to $6 \mathrm{PM}$. Therefore, the fuzzy expert system is developed in a way that DG operated between the above periods. The photovoltaic DG system under different power factor scenario is considered for investigation.

\section{Proposed Fuzzy Expert System}

The proposed fuzzy logic controller for DG integration implies Mamdani and Sugeno fuzzy inference system. The DG sizing is determined using Mamdani type fuzzy logic controller on the basis of feeder performance parameter such as substation reserve capacity, feeder power loss to load ratio, voltage unbalance, and apparent power imbalance, whereas Sugeno type fuzzy logic controller is used to choose the DG location on the basis of DG output, survivability index, and node distance from the substation. The fuzzybased expert system is tested using the MATLAB fuzzy logic tool box under multi-rules-based decision and multisets considerations as in Figure 1. 


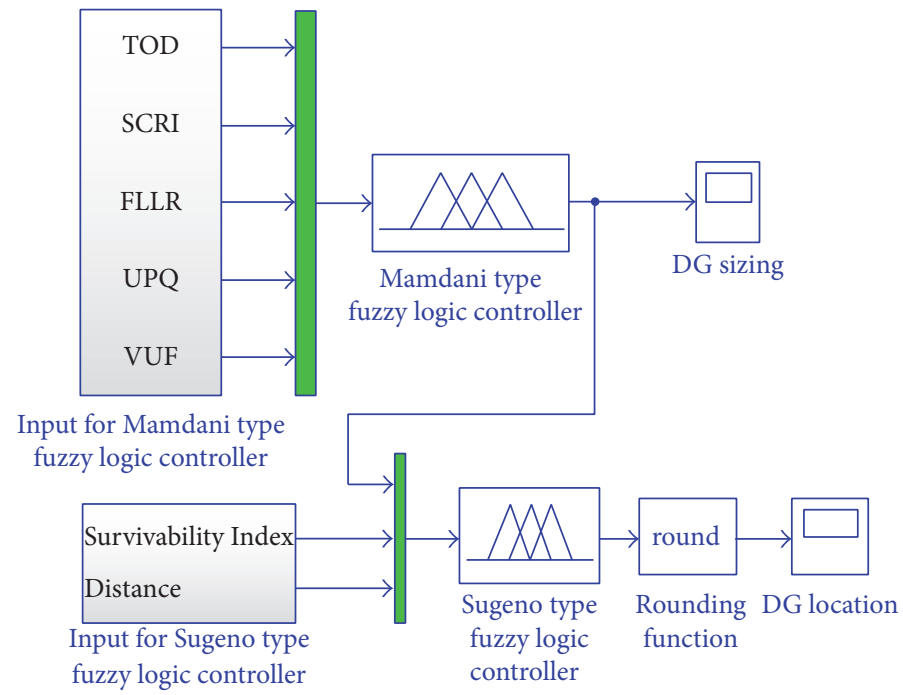

FIGURE 1: Fuzzy logic controller for DG integration.

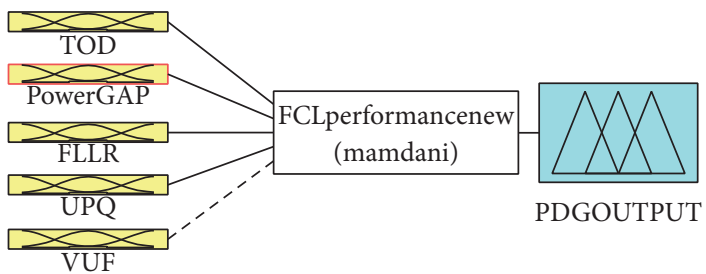

FIGURE 2: Mamdani type fuzzy logic controller for DG sizing.

Inference process for Mamdani type fuzzy logic controller has MIN-MAX method of aggregation and SOM of the defuzzification process as standard settings. The other setting for Sugeno type fuzzy logic controller remains the same except for defuzzification method named as whatever [16].

\subsection{Mamdani Type Fuzzy Logic Controller for DG Sizing.} Unbalance in voltage and current increased apparent power imbalance, feeder loss, voltage deviation, and neutral current. Increased neutral current in substation transformer leads to communication interference, equipment overloading, and false operation of the protective system. Apart from the above, apparent power imbalance is a more appropriate approach to reactive power compensation. The 15-minute characteristics time interval, substation reserve capacity (SRCI), feeder power loss to load ratio (FLLR), voltage unbalance factor (VUF), and apparent power imbalance (APBI) indices are the five inputs to the Mamdani type inference system, which are computed from the load flow solution over an each 15-minute time interval for the whole day [15]. Proposed Mamdani type inference as shown in Figure 2 has a set of 15 rules, which involve heuristic rules for determining the size of DG in the fuzzification process. In the fuzzification process, these inputs are converted into logic form in accordance with the associated membership functions.

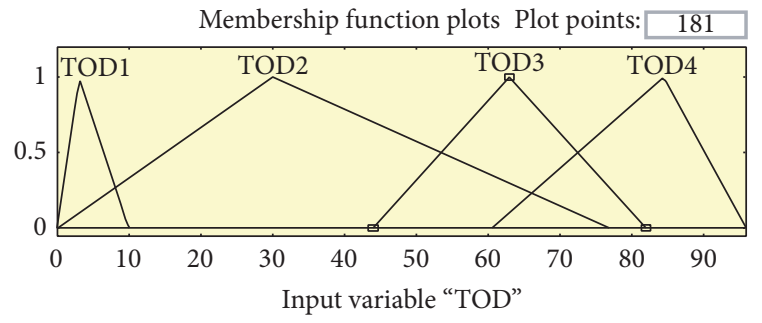

FIgURE 3: Membership function of TOD.

Four different times of day (TOD) in terms of 15-minute metering time interval is taken for the whole day and represented by four triangles membership function curves as shown in Figure 3. In Figure 4, the power demand gap is described by Z, Gauss, and S shape membership functions. The $Z$ shape membership function represents the demand gap less than $0.5 \mathrm{MW}$ and demand gap greater than $0.5 \mathrm{MW}$ is represented by an $S$ shape membership curve.

Triangle membership function is used for medium value FLLR, UPQ, and VUF, whereas trapezoidal membership functions are considered for the low and high of the above three variables. Their graphical representations of membership function are depicted in Figures 5, 6, and 7, respectively. 


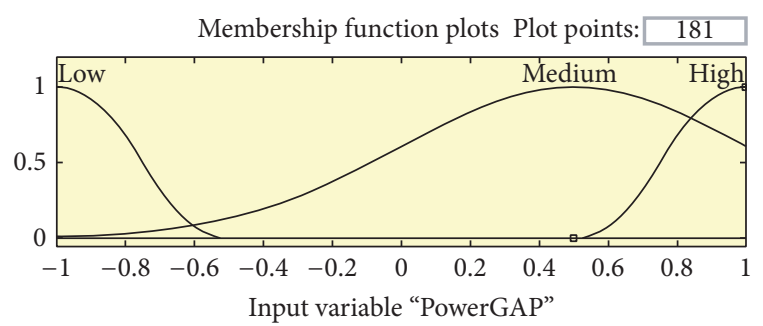

Figure 4: Membership function of power demand gap.

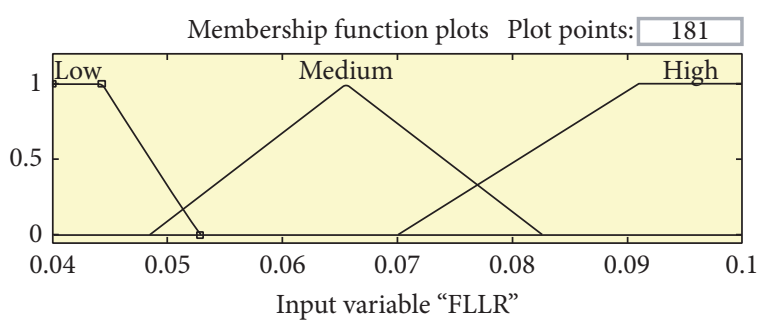

FIGURE 5: Membership function of FLLR.

DG sizing is shown with three trapezoidal membership functions in Figure 8. Low DG output is assumed to be between 0 and $0.1 \mathrm{MW}$, whereas medium DG output has ranged between 0.12 and 0.4 . The DG output is considered between 0.4 and $1 \mathrm{MW}$. Surface view of fuzzy logic controller rules for DG sizing is shown in Figure 9.

3.2. Sugeno Type Fuzzy Logic Controller for DG Location. This paper proposes a fuzzy approach to predict vulnerability of the node using survivability index. The proposed survivability index (SI) is computed by equation (2) below for each node using voltage stability margin (VSI) and voltage deviation index (VDI) corresponds to $90 \%$ percentile over each 15 minute time interval for the whole day $[14,15]$ :

$$
\mathrm{SI}=\min \left(\operatorname{VSI}_{a b c}^{h}\right) * 0.75+\max \left(\operatorname{VDI}_{a b c}^{h}\right) * 0.25 .
$$

Table 1 shows the survivability index of top 15 nodes from the list of nodes in or near vulnerability and Table 1 is depicted in the Appendix. The membership function for vulnerable nodes is represented in Figure 12.

Sugeno type fuzzy logic controller as shown in Figure 10 has crisp input parameters such as node distance from the substation, DG output, and vulnerable node. Node distance is depicted by three intersecting trapezoidal curves in Figure 11. The low distance corresponds to the range of 0 to 3300 feet and medium distance is between 3400 and 6000 feet. High distance is considered between 6000 and 8000 feet. The output of Sugeno type fuzzy logic controller has constant functions as shown in Figure 13, where the distributed generation can be added according to the demand from the customer premises. The proposed Sugeno type inference has a set of 09 rules to determine the location of DG as shown Figure 14.

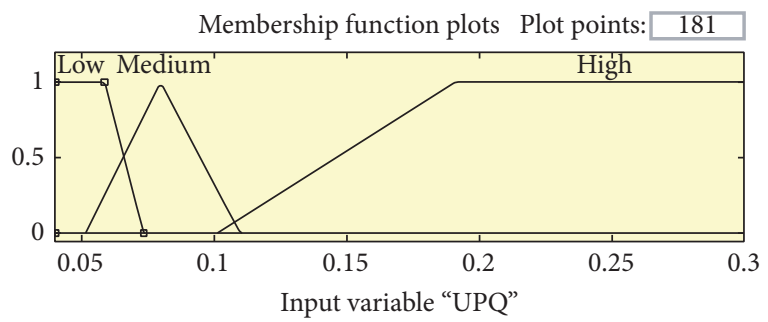

FIGURE 6: Membership function of UPQ.

TABLE 1: Survivability index of top 15 nodes.

\begin{tabular}{lccc}
\hline S. number & Node & Distance (Feet) & Survivability index \\
\hline 1 & 775 & 4930 & 0.881672 \\
2 & 709 & 4930 & 0.88071 \\
3 & 708 & 5250 & 0.889938 \\
4 & 733 & 5570 & 0.90528 \\
5 & 734 & 6130 & 0.918396 \\
6 & 737 & 6770 & 0.929216 \\
7 & 738 & 7170 & 0.93371 \\
8 & 711 & 7570 & 0.936115 \\
9 & 741 & 7970 & 0.936872 \\
10 & 732 & 5570 & 0.890948 \\
11 & 731 & 5530 & 0.880712 \\
12 & 710 & 6650 & 0.921796 \\
13 & 735 & 6850 & 0.923083 \\
14 & 736 & 7930 & 0.921934 \\
15 & 740 & 7770 & 0.937371 \\
\hline
\end{tabular}

Figures 15 and 16 show the inputs of Madami and Sugeno fuzzy logic controller, respectively, whereas outputs of Madami and Sugeno fuzzy logic controller are depicted in Figure 17. Node 734 is found at the optimum DG location with $440 \mathrm{KW}$ per Phase capacity to be operational between 37 and 73 characteristics time interval.

\section{Results and Analysis}

The proposed algorithm has been implemented in MATLAB and evaluated on modified unbalance IEEE 37 node test feeder. In this study, total optimum DG capacity of $440 \mathrm{KW}$ per phase is considered at node 734 , and the DG operating time interval very much resembles solar photovoltaic DG. In this paper, constant power factor DG model is considered and has constant $P_{\mathrm{dg}}$ active power at a pf $\mathrm{dg}$ constant power factor. To keep constant power factor, required $Q_{\mathrm{dg}}$ reactive power of the DG is computed by following equation [17]:

$$
Q_{\mathrm{dg}}=P_{\mathrm{dg}} \tan \left(\cos ^{-1}\left(P_{\mathrm{dg}}\right)\right) .
$$

In the base case, the performance indices are computed without any DG integration for the whole day. The base loss 


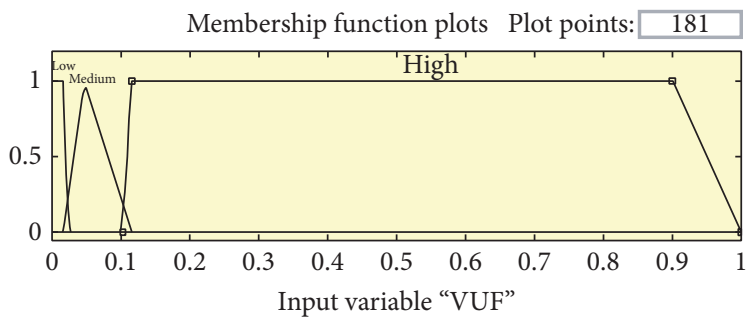

FIgURE 7: Membership function of VUF.

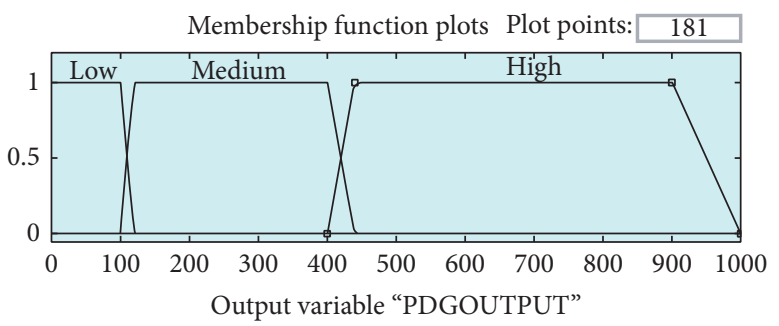

FIGURE 8: Membership function of DG output.

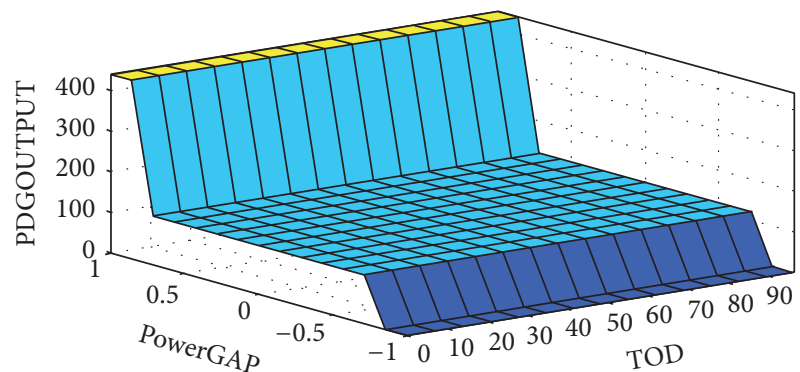

FIGURE 9: Surface view of fuzzy logic controller rules for DG sizing.

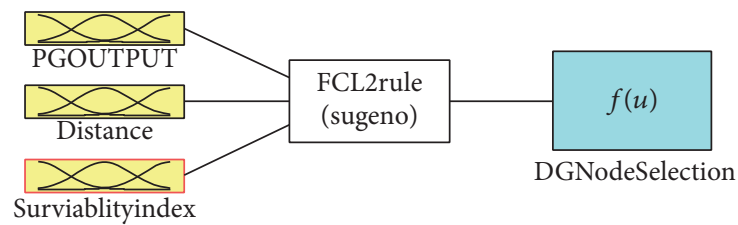

FIGURE 10: Sugeno type fuzzy logic controller for DG location.

of feeder is depicted in Figure 18. For the base case, the daily phase voltage profile for all buses is shown in Figures 19-21. The least voltage in Phase A, Phase- $B$, and Phase $C$ is found on nodes 34,33, and 31, respectively. These nodes can be used for shunt compensation.

The impacts of distributed generation on various indices are detailed as follows.

Figure 22 reveals that DG operation at 0.95 power factor leading has the highest substation reserve capacity, whereas

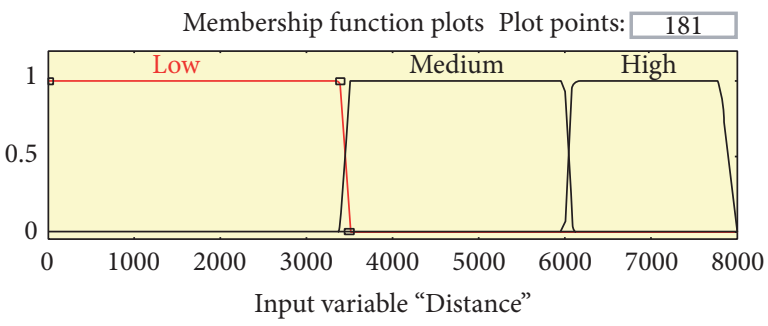

FIgURE 11: Membership function of distance.

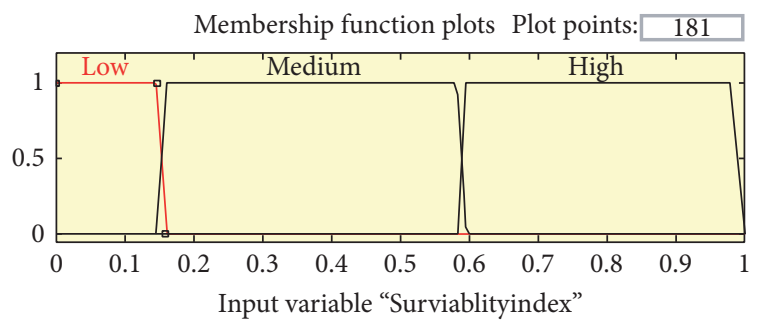

Figure 12: Membership function of survivability index.

\begin{tabular}{|c|c|}
\hline \multicolumn{2}{|c|}{ Membership function plots plot Points: $\square$} \\
\hline 775 & \\
\hline 709 & 741 \\
\hline 708 & 732 \\
\hline 733 & 731 \\
\hline 734 & 710 \\
\hline 737 & 735 \\
\hline 738 & 736 \\
\hline 711 & 740 \\
\hline
\end{tabular}

Output variable "DGNodeSelection"

FIgURE 13: Membership function of DG location. 


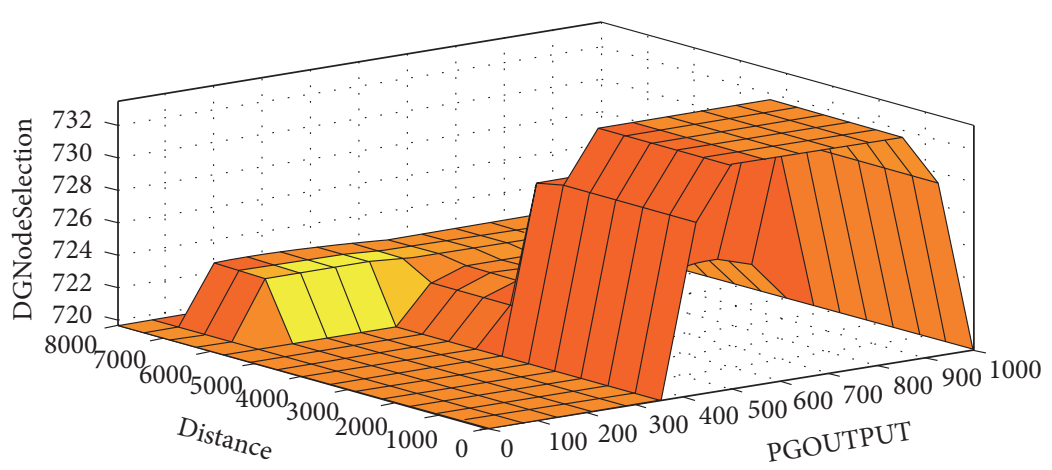

Figure 14: Surface view of Sugeno type fuzzy logic controller rules.
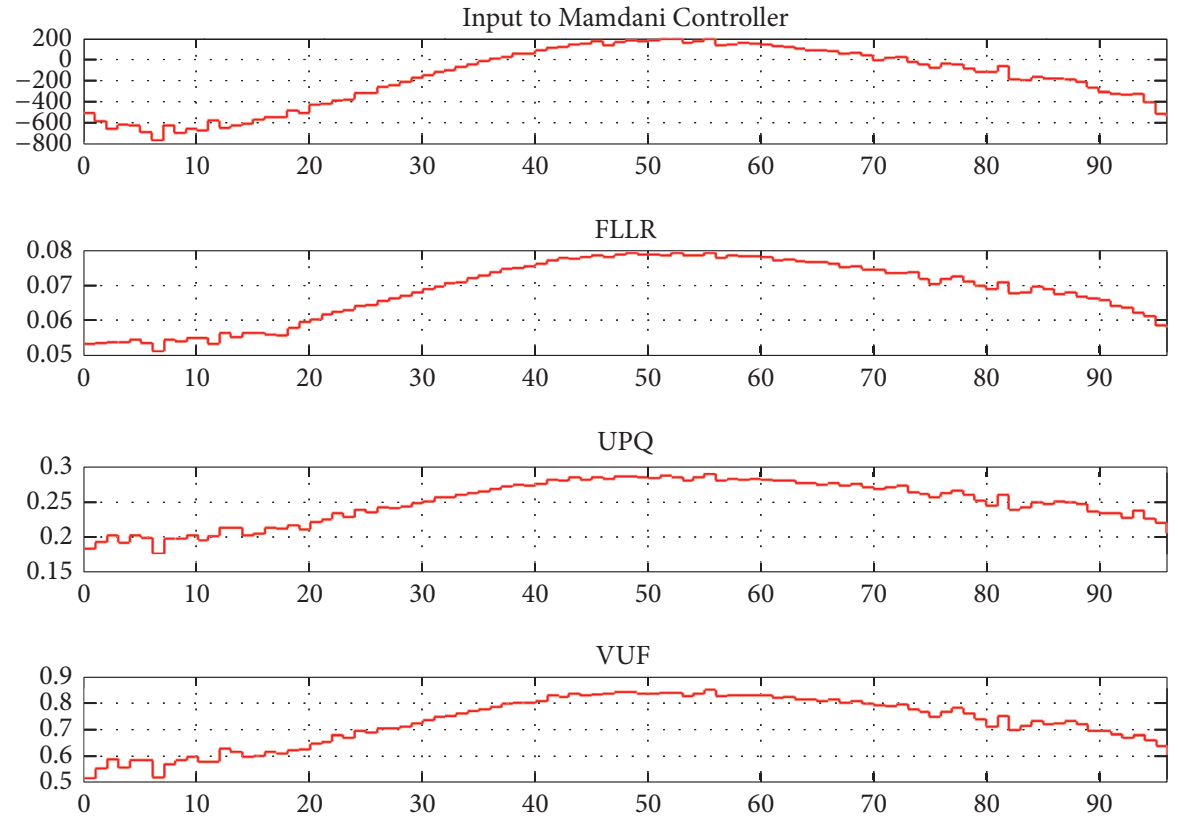

FIGURE 15: Inputs to Mamdani fuzzy logic controller.

the base case has the lowest substation reserve capacity between 35 and 71 characteristics time interval. DG operation at 0.95 lagging power factor has the reverse effect on load relief.

It is observed from Figures 23, 24, and 25 that the highest FLLR for all phases is found in DG operation at 0.95 power factor lag. Lowest reduction in FLLR in Phase-A, Phase-B, and Phase-C is found in DG 0.95 power factor (lag), base, and DG 0.99 power factor (lead) case, respectively.

In Figures 26, 27, and 28, it is observed that DG operation at 0.95 power factor leading showed highest BCLI reduction for all phases, the DG operation at 0.99 power factor leading got the second reduction in BCLI, and then the DG operation at unity power factor and DG operation at 0.95 power factor lagging the next. Base case showed a branch overloading
$1.173 \%$ and $1.15 \%$ at 53 and 55 characteristics time interval for Phase- $\mathrm{A}$ and $\mathrm{C}$, respectively.

As shown in Figure 29, there is no significant impact of DG placement over apparent power imbalance. Figures 30, 31 , and 32 reveal the same impact of DG placement on voltage deviation for all phases, which was observed in the case of BCLI.

As shown in Figures 33, 34, and 35, DG operation at 0.95 power factor lagging showed minimum power factor (MPF) for all phases. Maximum power factor for Phase-A and PhaseC occurred in the DG operation at 0.95 power factor leading, while maximum power factor for Phase-B occurred in DG operation at unity power factor.

In Figure 36, base case showed a highest voltage unbalance factor (VUF), the DG operation at 0.95 power factor 


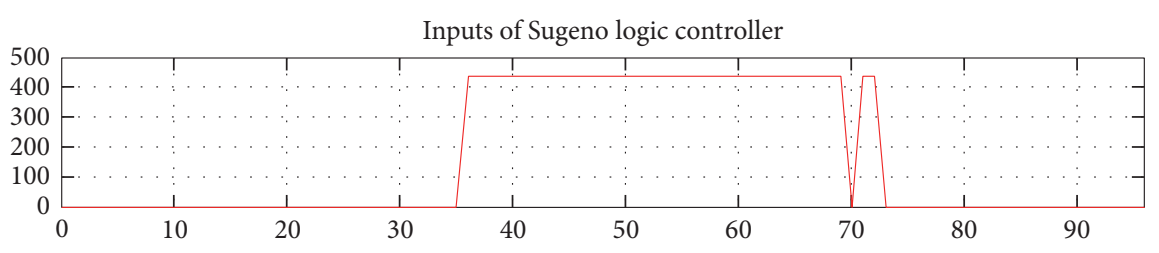

— Per Phase DG Capacity

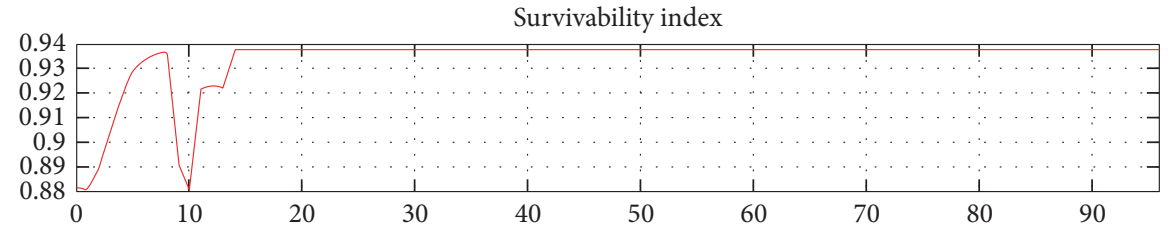

_ Survivability index

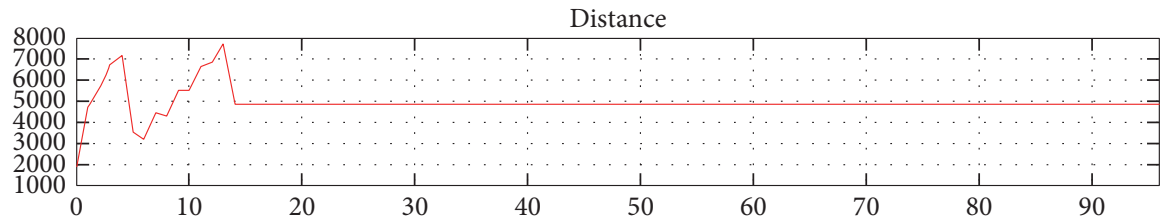

_ Distance

FIgURE 16: Inputs to Sugeno fuzzy controller.

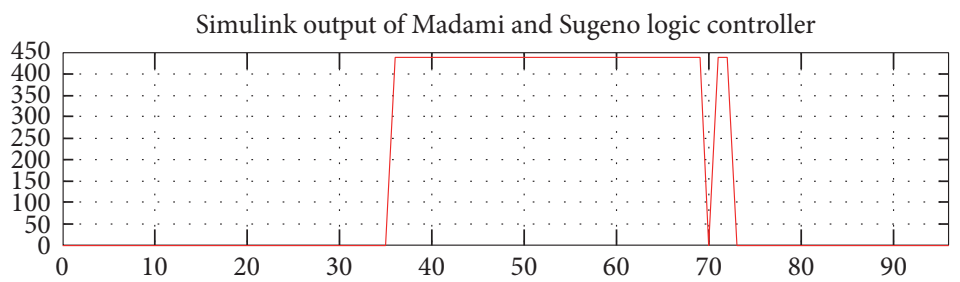

_ Per Phase DG Capacity

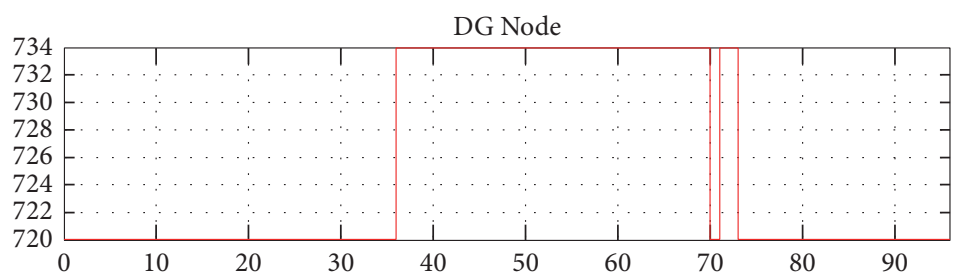

$\lceil$ DG Node

FIGURE 17: Simulink outputs of fuzzy logic controller.

lagging got the second maximum VUF, and then the DG operation at unity power factor and DG operation at 0.99 power factor leading the next. DG operation at 0.95 power factor lagging showed a maximum VUF of 0.5177 at 6 characteristics time interval.

\section{Conclusion}

The stochastic behavior of DG integrated distribution feeder is a challenging problem for operations and planning aspects. A fuzzy expert system is proposed to determine optimal 


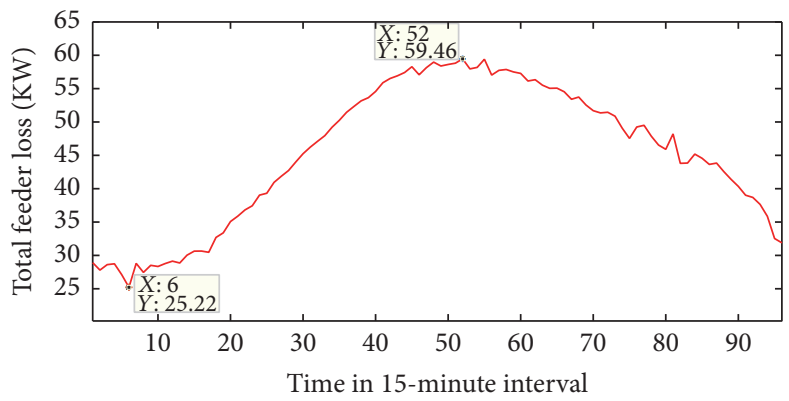

Figure 18: Feeder loss for base case.

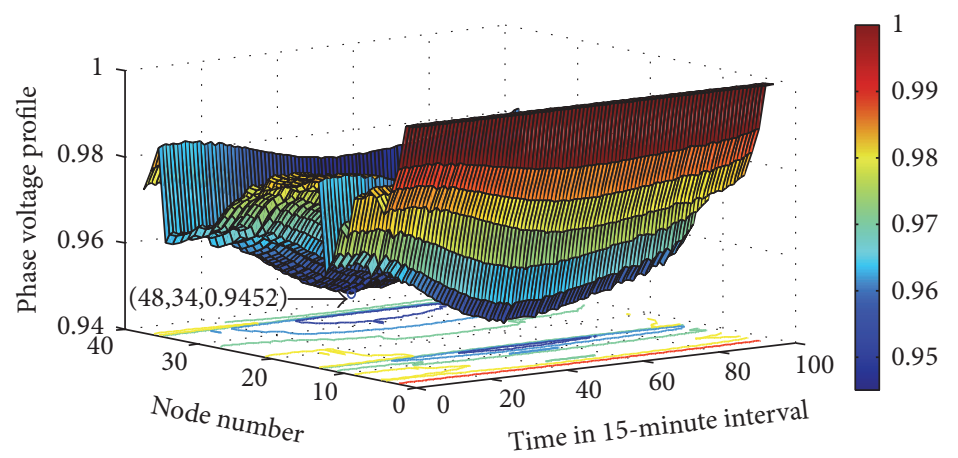

FIgUre 19: Phase voltage profile of Phase-A.

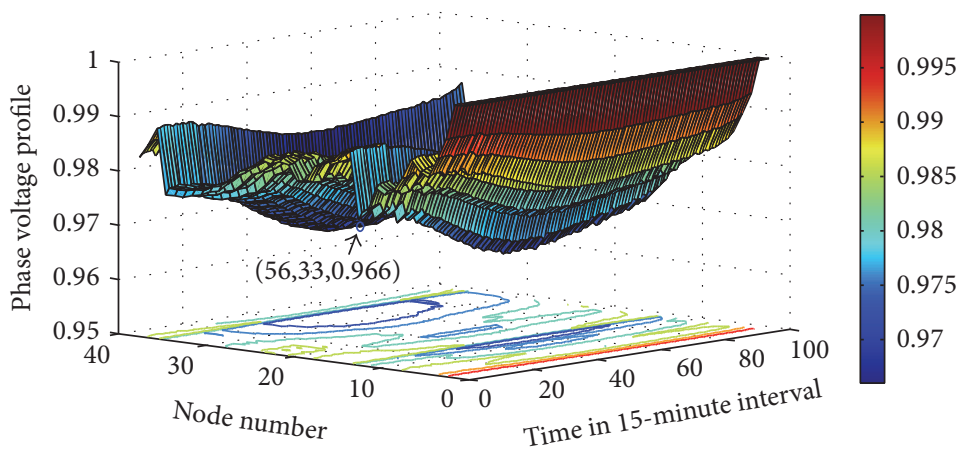

FIgure 20: Phase voltage profile of Phase-B.

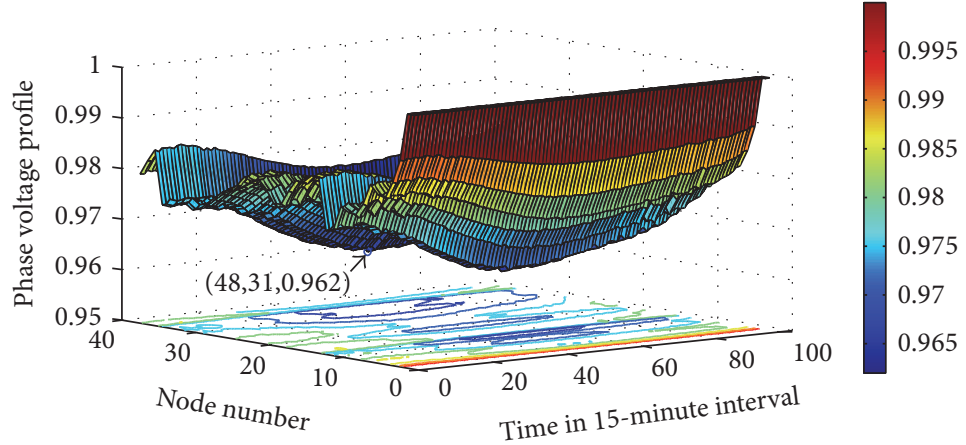

FIgURE 21: Phase voltage profile of Phase-C. 


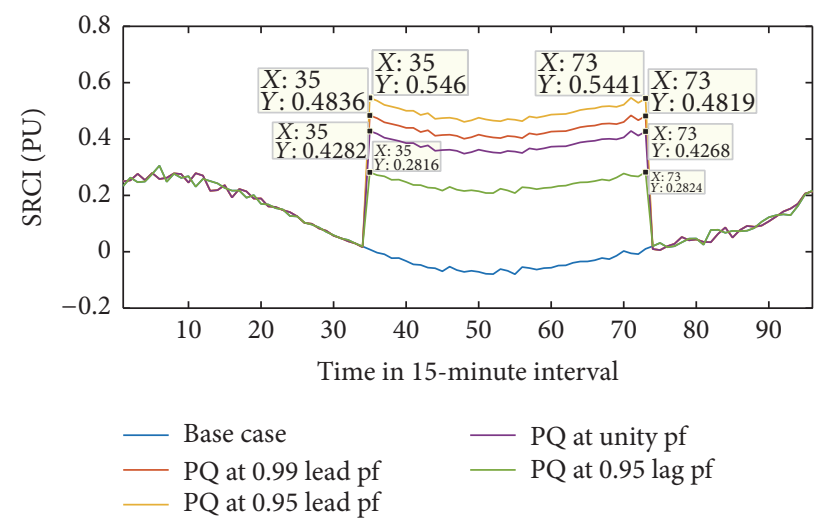

FIGURE 22: DG impact of substation reserve capacity index.

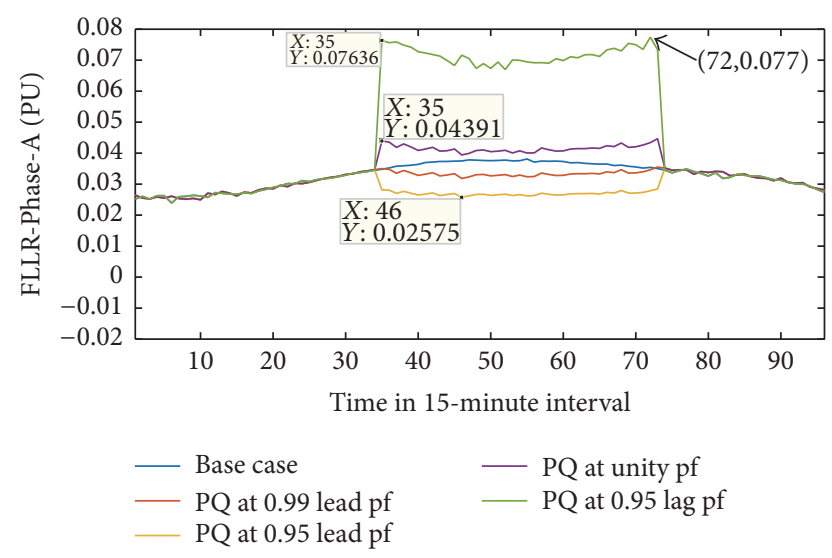

Figure 23: DG impact of feeder loss to load ratio for Phase-A.

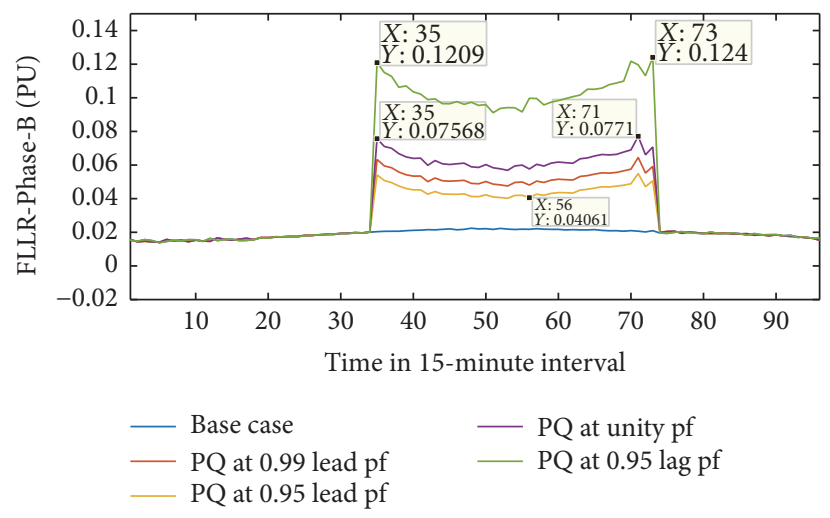

Figure 24: DG impact of feeder loss to load ratio for Phase-B.

location and size of fixed PQ DG integration to a modified unbalance IEEE 37 feeder. In this paper, impact of DG integration on performance indices has been demonstrated under at different power factor under stochastic environment. To investigate clear effect of DG operation for making

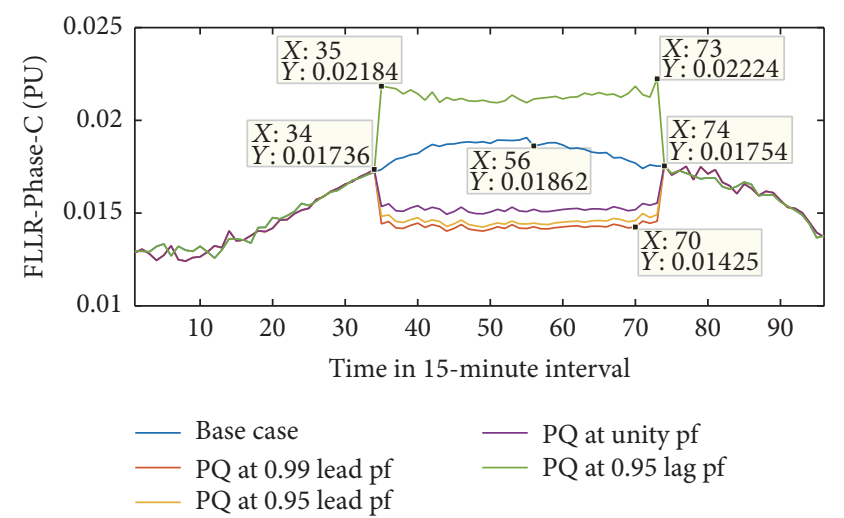

FIGURE 25: DG impact of feeder loss to load ratio for Phase-C.

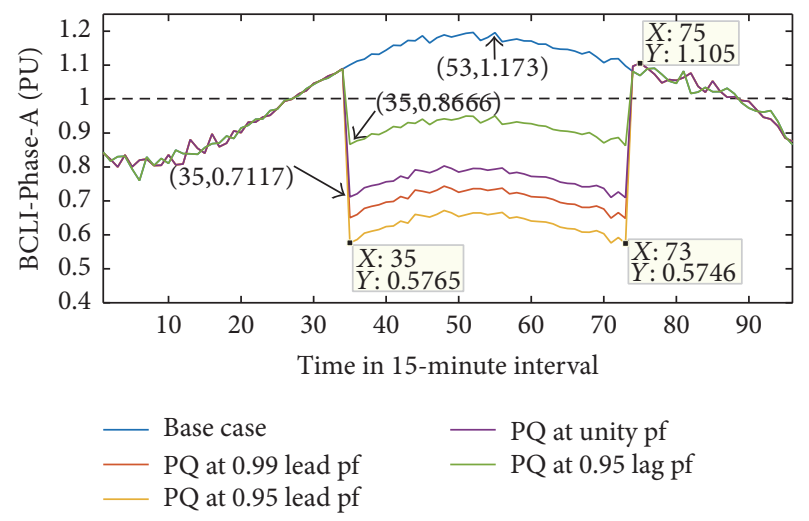

FIgURE 26: DG impact on branch loading index of Phase-A.

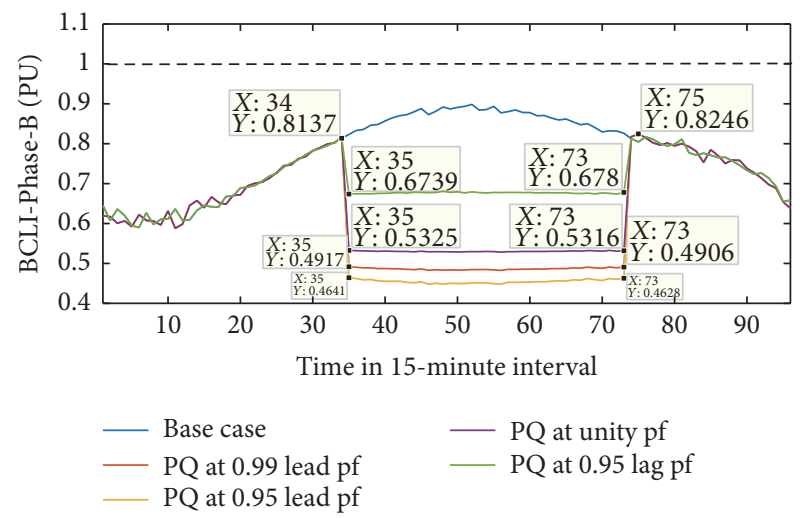

FIgURE 27: DG impact on branch loading index of Phase-B.

useful decision support, voltage regulator of the above feeder is removed. Comparative results obtained with different fixed PQ DG operating scenario are discussed and it is found that the results obtained by DG operation at 0.95 power factor leading are more suitable to improve performance of feeder. 

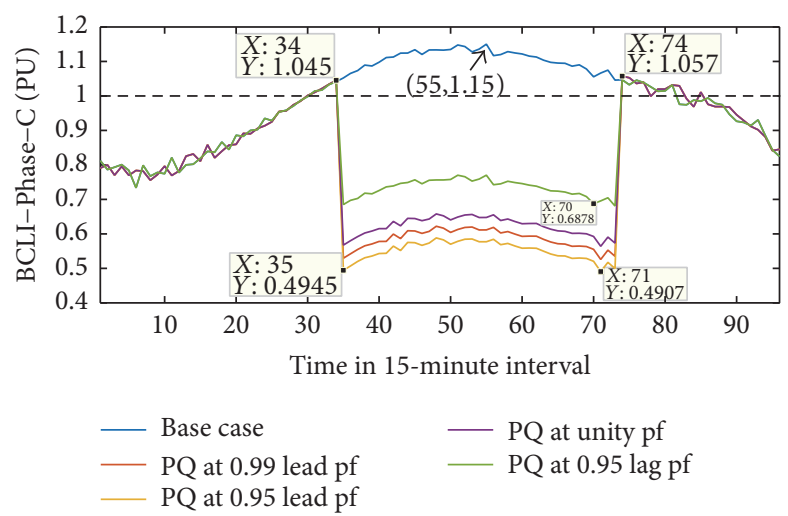

FIGURE 28: DG impact on branch loading index of Phase-C.

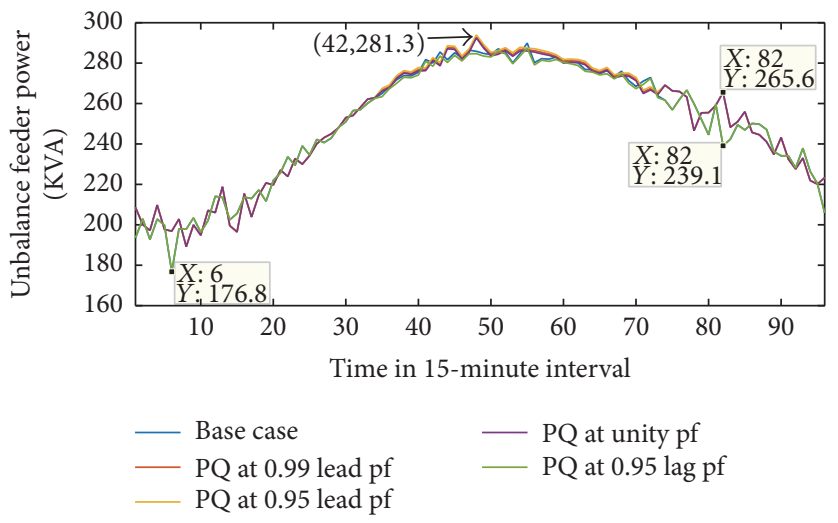

FIGURE 29: DG impact on apparent powers unbalance index of feeder.

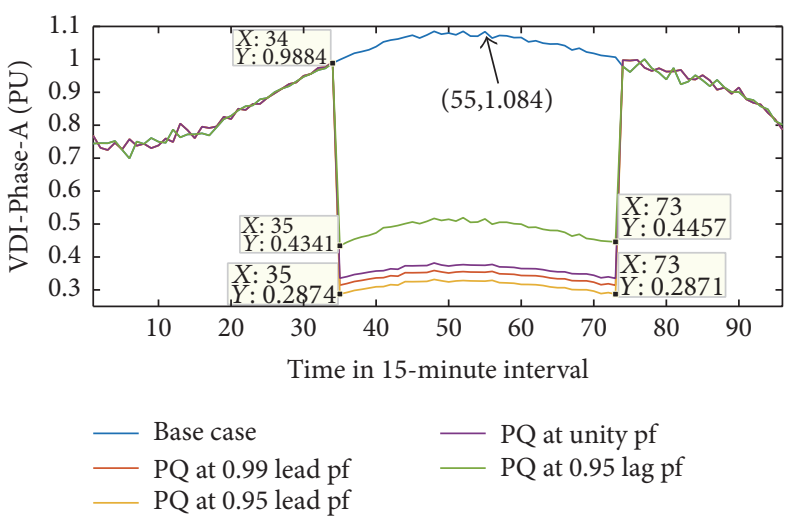

FIGURE 30: DG impact on maximum voltage deviation, Phase-A.

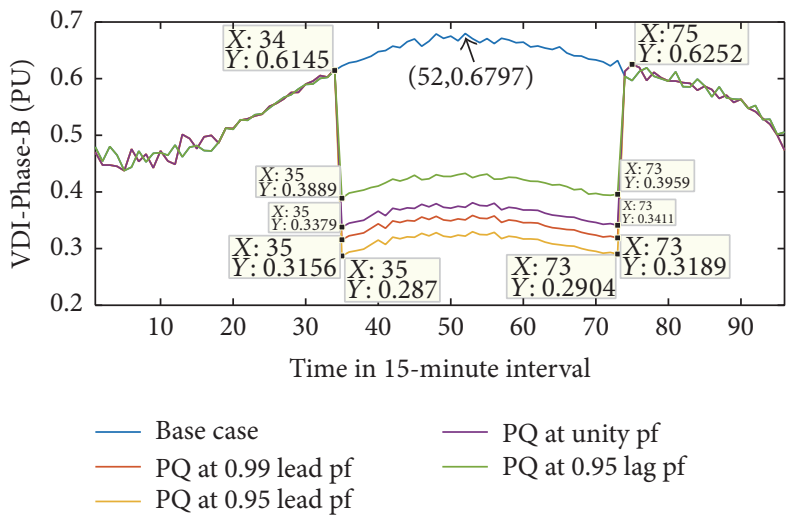

FIGURE 31: DG impact on maximum voltage deviation, Phase-B. 


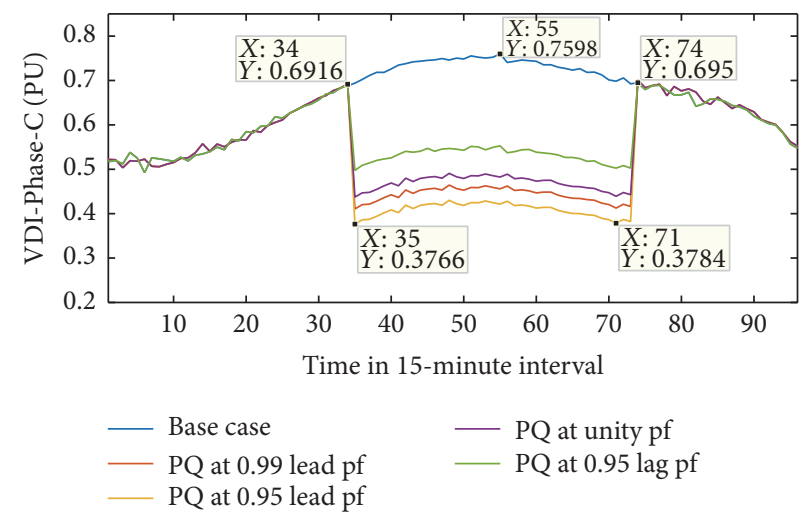

Figure 32: DG impact on maximum voltage deviation, Phase-C.

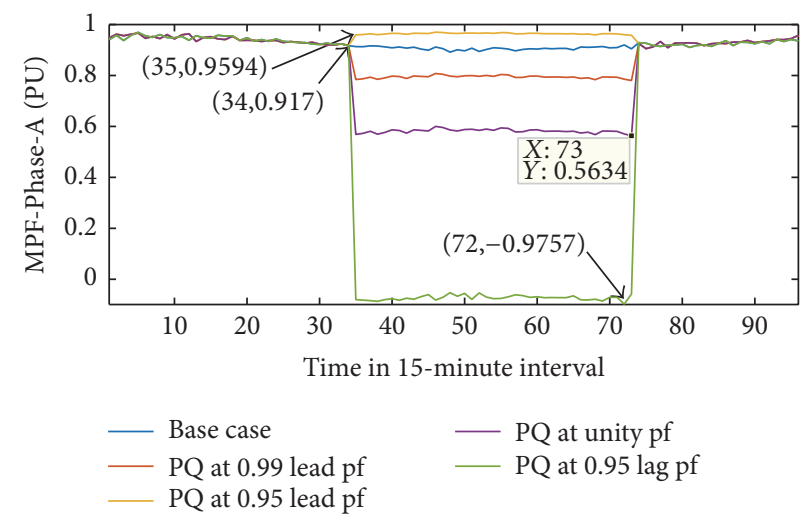

Figure 33: DG impact on minimum power factor of Phase-A.

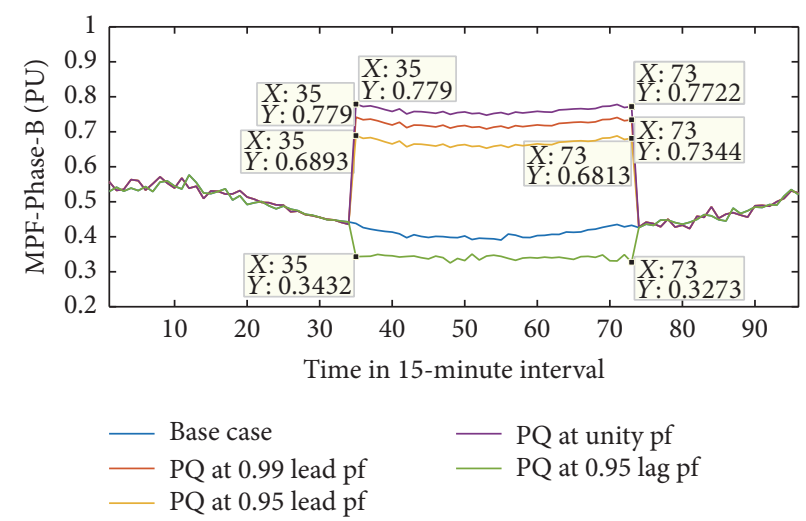

FIGURE 34: DG impact on minimum power factor of Phase-B.

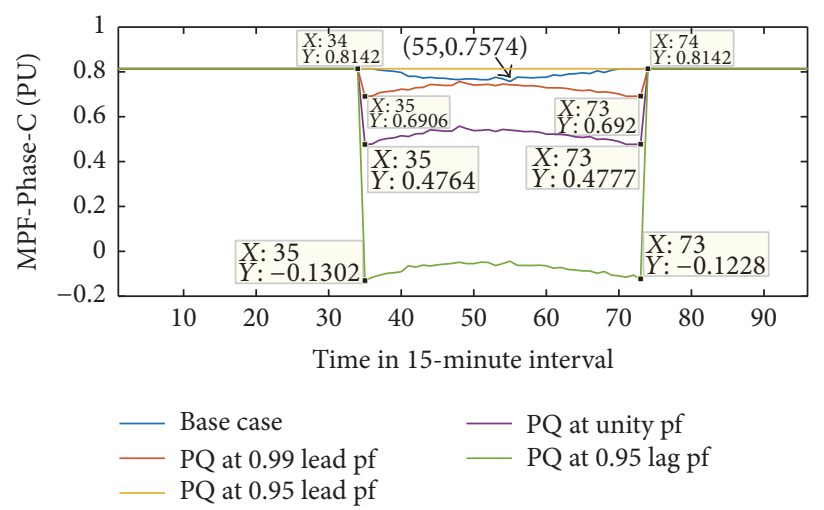

FIgURE 35: DG impact of minimum power factor of Phase-C. 


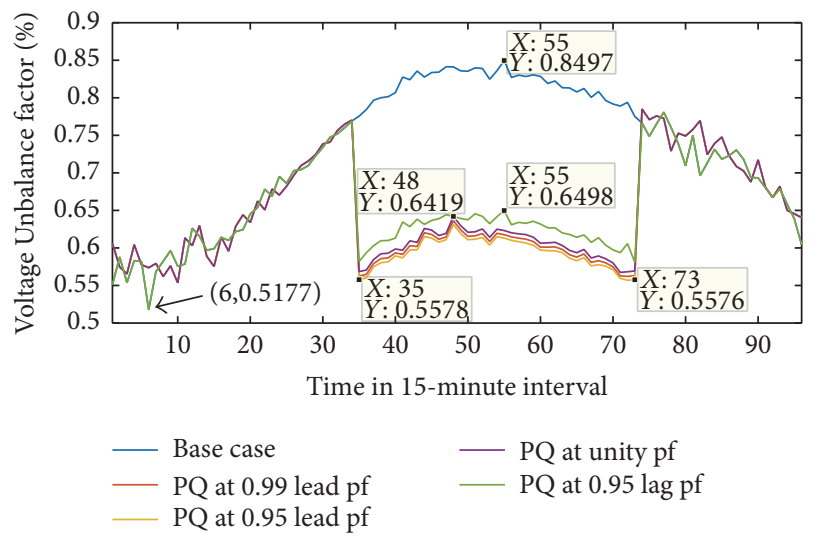

FIGURE 36: DG impact of voltage unbalance factor of feeder.

\section{Appendix}

\section{A. Survivability Index of Top 15 Nodes}

(see Table 1).

\section{B. ZIP Load Compositions Calculation}

To find ZIP load composition at each bus $k$, the following equations were utilized to compute ZIP load composition at each bus $k$ for each $h$ characteristics time interval:

$$
\begin{aligned}
C 1_{a b c}^{k}(h)= & 0.8 * P I_{a b c}^{k}(h)+0.6 * P C_{a b c}^{k}(h)+0.8 \\
& * P R_{a b c}^{k}(h), \\
C 2_{a b c}^{k}(h)= & 0.2 * P I_{a b c}^{k}(h)+0.4 * P C_{a b c}^{k}(h)+0.19 \\
& * P R_{a b c}^{k}(h), \\
C 3_{a b c}^{k}(h)= & * P I_{a b c}^{k}(h)+0 * P C_{a b c}^{k}(h)+0.01 \\
& * P R_{a b c}^{k}(h), \\
D 1_{a b c}^{k}(h)= & 0.8 * Q I_{a b c}^{k}(h)+0.6 * Q C_{a b c}^{k}(h)+0.8 \\
& * Q R_{a b c}^{k}(h), \\
D 2_{a b c}^{k}(h)= & 0.2 * Q I_{a b c}^{k}(h)+0.4 * Q C_{a b c}^{k}(h)+0.19 \\
& * Q R_{a b c}^{k}(h), \\
D 3_{a b c}^{k}(h)= & * Q I_{a b c}^{k}(h)+0 * Q C_{a b c}^{k}(h)+0.01 \\
& * Q R_{a b c}^{k}(h) .
\end{aligned}
$$

\section{Nomenclature}

$P_{a b c}^{k}(h), Q_{a b c}^{k}(h)$ : Total real and reactive power load at bus $k$ during $h$

$V N_{a b c}, V_{a b c}^{k}(h)$ : Nominal and three-phase voltage at $k$ node
$C 1_{a b c}^{k}(h), C 2_{a b c}^{k}(h), C 3_{a b c}^{k}(h):$ ZIP active load composition at bus $k$ during $h$

$D 1_{a b c}^{k}(h), D 2_{a b c}^{k}(h), D 3_{a b c}^{k}(h)$ : ZIP reactive load composition at bus $k$ during $h$

$P I_{a b c}^{k}(h), P R_{a b c}^{k}(h), P C_{a b c}^{k}(h):$ Active power for industrial, residential, and commercial load connected at bus $k$ during $h$

$Q I_{a b c}^{k}(h), Q R_{a b c}^{k}(h), Q C_{a b c}^{k}(h)$ : Reactive power for industrial, residential, and commercial load connected at bus $k$ during $h$

$h:$ 15-minute characteristics time interval.

\section{Competing Interests}

The authors declare that there is no conflict of interests regarding the publication of this paper.

\section{References}

[1] A. Barin, C. U. Brazil, L. Martins, and E. S. A. Brazil, "Fuzzy based expert system for renewable energy management," in Proceedings of the CIRED Workshop, no. 0020, Rome, Italy, 2014.

[2] N. Hashmi and S. A. Khan, "Power energy management for a grid-connected PV system using rule-base fuzzy logic," in Proceedings of the 3rd International Conference on Artificial Intelligence, Modelling \& Simulation (AIMS '15), pp. 31-36, Kota Kinabalu, Malaysia, December 2015.

[3] A. R. Kashfi and M. E. El-Hawary, "Integration of distributed generation in medium voltage distribution network using fuzzy logic controller for demand side management," in Proceedings of the Electrical Power and Energy Conference (EPEC '14), pp. 254-259, Calgary, Canada, November 2014.

[4] S. K. Injeti and N. P. Kumar, "Planning and operation of active radial distribution networks for improved voltage stability and loss reduction," World Journal of Modelling and Simulation, vol. 8, no. 3, pp. 211-222, 2012. 
[5] Y. Manjili and A. Rajaee, "Fuzzy Control of Electricity Storage Unit for Energy Management of Micro-Grids," http://ace.wacong.org/Assets/doc/Fuzzy\%20Control\%20of\%20Electricity\%20 Storage $\% 20$ Unit $\% 20$ for\%20Energy\%20Management $\% 20$ of $\%$ 20Micro-Grids_Mexico\%20WAC\%202012.pdf.

[6] A. Metia and S. Ghosh, "Fuzzy based DG allocation for Loss Minimization in a Radial Distribution System," Balkan Journal of Electrical \& Computer Engineering, vol. 3, no. 3, pp. 115-123, 2015.

[7] E. A. Mohamed, M. M. Othman, and Y. G. Hegazy, "Optimal sizing and placement of distributed generators for profit maximization using firefly algorithm," in Proceedings of the International Conference on Artificial Intelligence, Energy and Manufacturing Engineering (ICAEME '14), pp. 6-10, 2014.

[8] K. Muthukumar and S. Jayalalitha, "Optimal reactive power compensation by shunt capacitor sizing using harmony search algorithm in unbalanced radial distribution system for power loss minimization," International Journal on Electrical Engineering and Informatics, vol. 5, no. 4, pp. 474-491, 2013.

[9] M. Padma Lalitha, V. C. Veera Reddy, and N. Sivarami Reddy, "Application of fuzzy and ABC algorithm for DG placement for minimum loss in radial distribution system," Iranian Journal of Electrical and Electronic Engineering, vol. 6, no. 4, pp. 248-257, 2010.

[10] M. S. Thomas, R. Ranjan, and R. Roma, "Probabilistic fuzzy approach to assess RDS vulnerability and plan corrective action using feeder reconfiguration," Energy and Power Engineering, vol. 4, no. 5, pp. 330-338, 2012.

[11] P. N. Si and S. S. Win, "Fuzzy algorithm for capacitor allocation and sizing in radial distribution system to reduce losses," International Journal of Science, Engineering and Technology Research, vol. 3, no. 12, 2014.

[12] A. Arabali, M. Ghofrani, M. Etezadi-Amoli, and M. S. Fadali, "Stochastic performance assessment and sizing for a hybrid power system of Solar/Wind/Energy Storage," IEEE Transactions on Sustainable Energy, vol. 5, no. 2, pp. 363-371, 2014.

[13] A. Soroudi and M. Ehsan, "A possibilistic-probabilistic tool for evaluating the impact of stochastic renewable and controllable power generation on energy losses in distribution networks-a case study," Renewable and Sustainable Energy Reviews, vol. 15, no. 1, pp. 794-800, 2011.

[14] J. P. Sharma and H. Ravishankar Kamath, "Stochastic voltage assessment of unbalance radial feeder," Journal of Electrical Systems, vol. 11, no. 3, pp. 258-270, 2015.

[15] J. P. Sharma and H. R. Kamath, "Performance analysis of unbalance radial feeder with time varying composite load," Journal of Power and Energy Engineering, vol. 3, no. 5, pp. 56-70, 2015.

[16] The MathWorks Inc, Types of Fuzzy Interefernece Systems, The MathWorks Inc, 1994, 1994-2014, http://www.mathworks.com/ help/fuzzy/types-of-fuzzyinference-systems.html.

[17] J.-H. Teng, "Modelling distributed generations in three-phase distribution load flow," IET Generation, Transmission and Distribution, vol. 2, no. 3, pp. 330-340, 2008. 


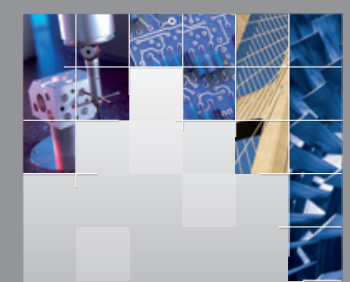

\section{Enfincering}
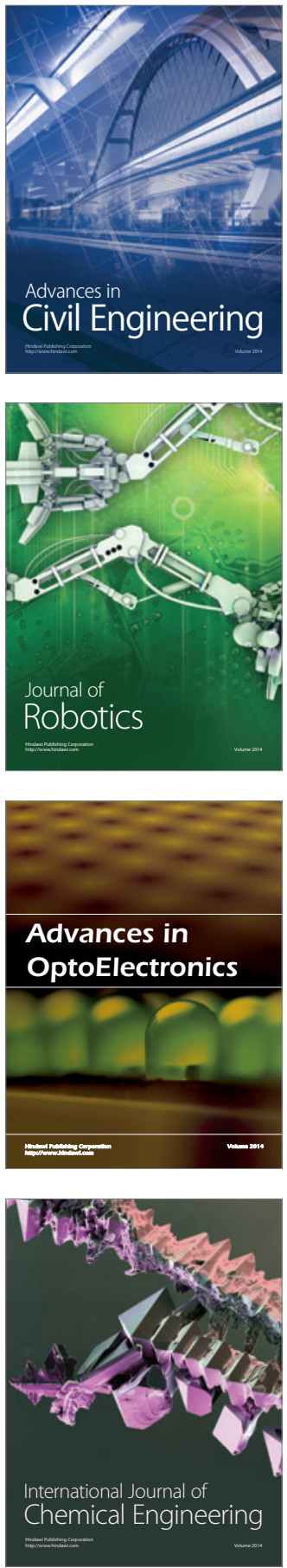

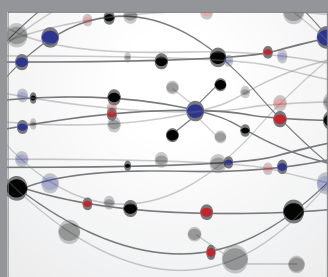

The Scientific World Journal

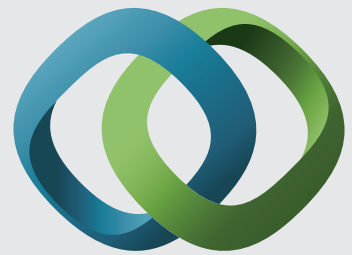

\section{Hindawi}

Submit your manuscripts at

http://www.hindawi.com
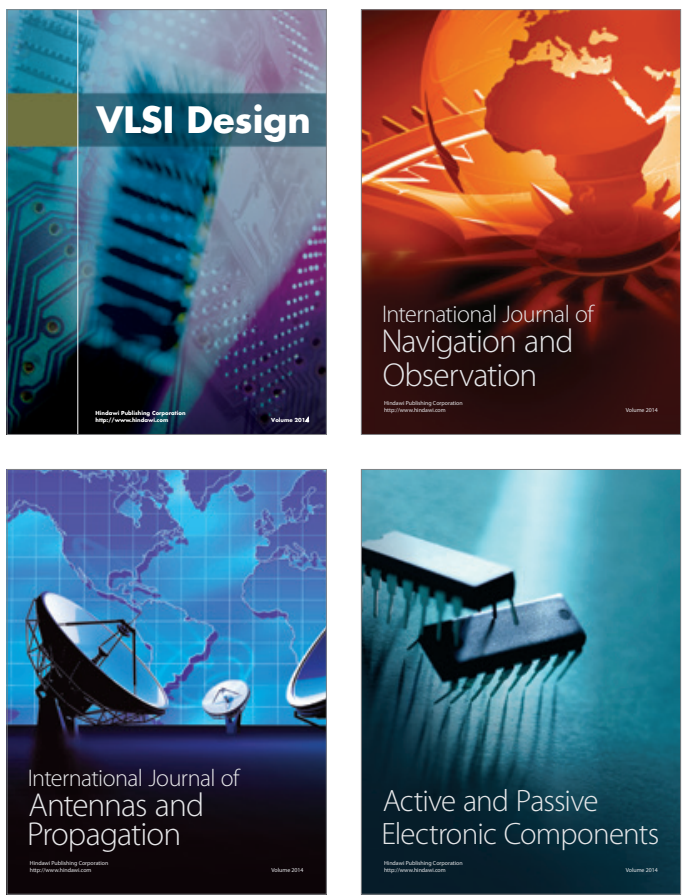
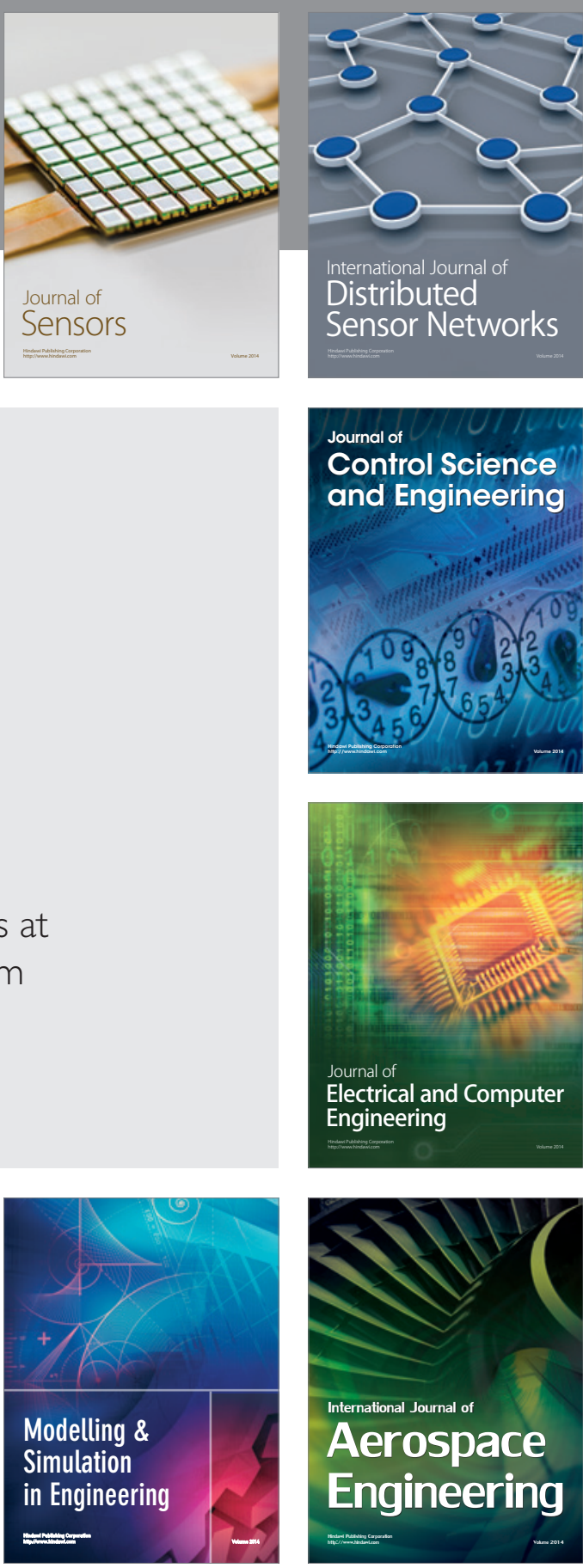

International Journal of

Distributed

Sensor Networks

Journal of

Control Science

and Engineering
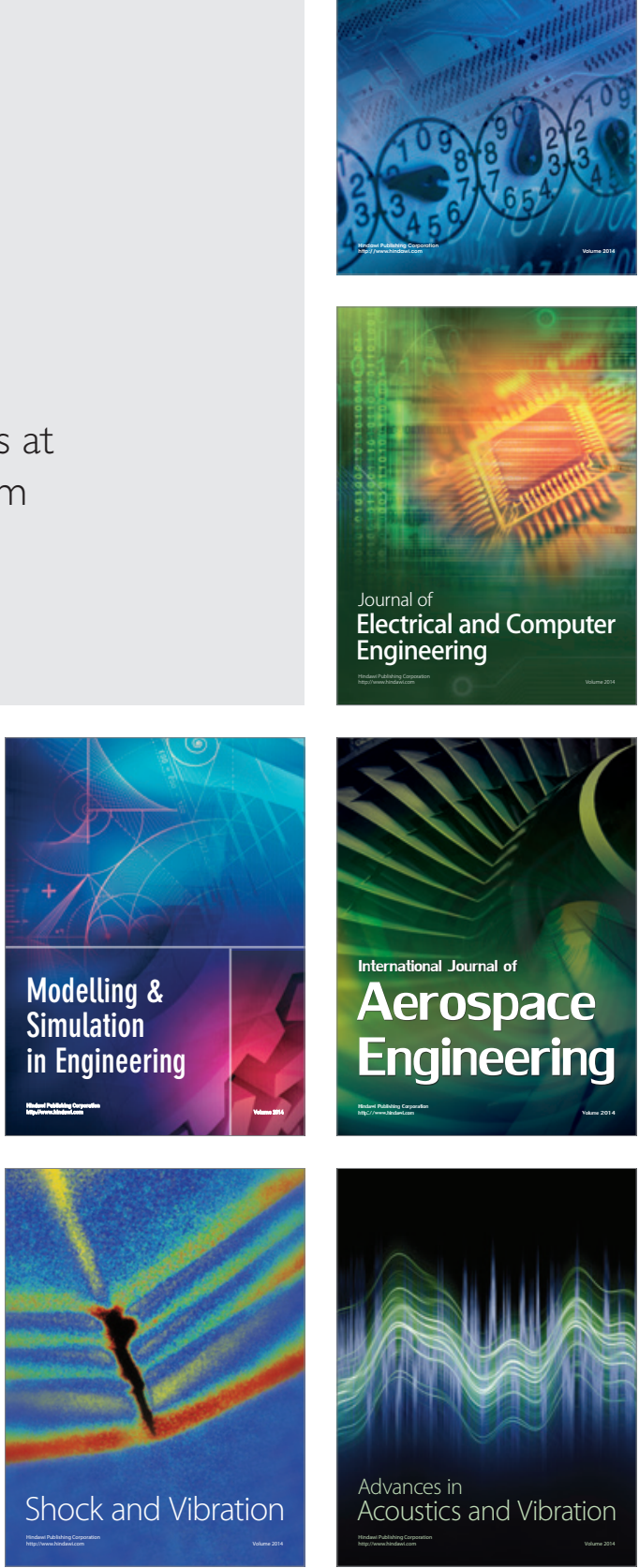